\title{
Monte-Carlo simulation of graphene in terms of occupation numbers for the excitonic order parameter at hexagonal lattice.
}

\author{
Oleg Pavlovsky ${ }^{* \dagger}$ \\ Institute for Theoretical Problems of Microphysics, Moscow State University and Institute for \\ Theoretical and Experimental Physics, Moscow, Russia \\ E-mail: ovpegoa.bog.msu.ru
}

\author{
Anna Sinelnikova \\ Institute for Theoretical Problems of Microphysics, Moscow State University and Institute for \\ Theoretical and Experimental Physics, Moscow, Russia \\ E-mail: sinelegoa.bog.msu.ru

\section{Maxim Ulybyshev} \\ Institute for Theoretical Problems of Microphysics, Moscow State University and Institute for \\ Theoretical and Experimental Physics, Moscow, Russia \\ E-mail: uLybyshev1dgoa.bog.msu.ru
}

\begin{abstract}
We present the results of the Monte-Carlo simulation of graphene-like statistical model in terms of occupation numbers. We study the problem of the phase transition in graphene to an insulating phase. Only antiferromagnetic order parameter was studied at the moment by means of Hybrid Monte-Carlo process on the hexagonal lattice because of the sign problem for excitonic order parameter in fermionic determinant. Therefore we have studied the possibility of the phase transition to an excitonic phase by means of simplified Monte-Carlo process on the hexagonal lattice. We show that this phase, which corresponds to a charge polarization of graphene sublattices, can appear at some values of Coulomb electron-electron potentials. In this work we study the dependence of the phase transition characteristics on the strength of on-site electron-electron interaction. We show that excitonic phase transition is also impossible in suspended graphene.
\end{abstract}

31st International Symposium on Lattice Field Theory - LATTICE 2013

July 29 - August 3, 2013

Mainz, Germany

\footnotetext{
* Speaker.

${ }^{\dagger}$ Numerical calculations were performed at Supercomputing Center of Moscow State University
} 


\section{Introduction}

One of the most essential problems in graphene physics is a problem of electronic transport. Electronic excitations in graphene strongly interact with each other. The strength of the interaction is controlled by the substrate dielectric permittivity so the strongest interaction is in suspended graphene. The conductivity of suspended graphene has been an open problem for many years. Many theoretical models [ [, , [2] have predicted the existence of the mass gap in the case of free graphene sheet which appears due to spontaneous breaking of sublattice symmetry. This theoretical prediction have been verified numerically within the framework of effective field theory of graphene [ [ [ , 目, []]. Resent experimental study [6] of suspended graphene have shown that this material remains in metallic state even without any substrate. This discrepancy was solved in the paper [ [ $[$ ]. It was shown that if one takes into the account the screening of the Coulomb potential at short distances, the tight-binding model of graphene predicts the semimetal state for suspended graphene.

There are several order parameters and possible fermion condensates which were discussed in

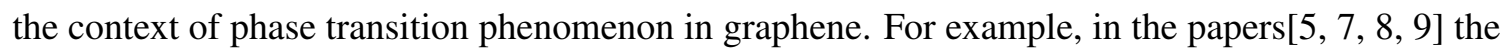
effect of spontaneous polarization of spins was studied. From this point of view, the dielectric phase is an antiferromagnetic state. The another possibility is excitonic condensation broadly discussed both in theoretical papers and lattice simulations by means of effective graphene field model. From microscopic point of view, two sublattices of the hexagonal lattice acquire opposite charges in this phase.

It is also important to emphasize that the study of this excitonic order parameter within the framework of Hybrid Monte-Carlo simulations (like it was done in [ $[\mathbf{}]$ and [ $[9]$ for the antiferromagnetic order parameter) is impossible now due to the sign problem in fermionic determinant. So in this paper we have used simplified Monte Carlo method formulated in terms of occupation numbers where this problem doesn't arise.

\section{Graphene model in terms of occupation numbers}

Let us consider an ideal monolayer graphene. Carbon atoms in it form two dimensional hexagonal lattice. There is a 4-valency carbon atom in every site of this lattice. Three electrons of each carbon atom participate in chemical bonding ( $\sigma$-bound) with the neighboring atoms and the fourth electron ( $\pi$-orbital) causes the conductivity of graphene. We use the following Hamiltonian to describe the electric properties of this material [ए]]:

$$
H=H_{\kappa}+H_{H}+H_{C}=-\kappa \sum_{x, \rho_{b}, \sigma}\left(\psi_{\sigma, x+\rho_{b}}^{\dagger} \psi_{\sigma, x}+\text { h.c. }\right)+\frac{1}{2} \sum_{x} q_{x} V_{x, x} q_{x}+\frac{1}{2} \sum_{x \neq x^{\prime}} q_{x} V_{x, x^{\prime}} q_{x^{\prime}} .
$$

Different terms of Hamiltonian correspond to different physical phenomena. $H_{\kappa}$ corresponds to hoppings from one site to the nearest neighboring ones. $\psi_{\sigma, x}^{\dagger}$ and $\psi_{\sigma, x}$ are the creation and annihilation operators for the electrons at the site $x$ and up or down spin, $\rho_{b}, b=1,2,3$ are the vectors between the site $x$ and three its neighbors. The term $H_{H}$ is the on-site Coulomb interaction, $q_{x}$ is the operator of electrical charge for the site $x$ :

$$
q_{x}=1-\psi_{\uparrow, x}^{\dagger} \psi_{\uparrow, x}-\psi_{\downarrow, x}^{\dagger} \psi_{\downarrow, x} .
$$


"One" in this formula represents the fact that the charge of the lattice site is equal to 1 in the absence of electrons on $\pi$-orbitals. Finally, the last term in (1) is a Coulomb interaction of electrons at different sites of the lattice. $V_{x, x^{\prime}}$ is a Coulomb potential screened by $\sigma$-orbitals at short distances. We will discuss this phenomenon more properly in the next part of the paper.

It's convenient to introduce electron and hole excitations. Let us consider the creation and annihilation operators for the "electrons" ( $a$ operators) and "holes" ( $b$ operators) at the site $x$ :

$$
a_{x}^{\dagger}=\psi_{x, \uparrow}^{\dagger}, a_{x}=\psi_{x, \uparrow}, b_{x}^{\dagger}=\psi_{x, \downarrow}, b_{x}=\psi_{x, \downarrow}^{\dagger} .
$$

The charge operator in terms of operators $a_{x}$ and $b_{x}$ can be expressed as:

$$
q_{x}=1-a_{x}^{\dagger} a_{x}-b_{x} b_{x}^{\dagger}=b_{x}^{\dagger} b_{x}-a_{x}^{\dagger} a_{x} .
$$

There are four different states for every site $x$ :

1) The state $|\cdot\rangle$. There is no electrons on the $\pi$-orbital.

2) The states $|\uparrow \cdot\rangle$ and $|\downarrow \cdot\rangle$. There is only one electron on the $\pi$-orbital.

3) The state $|\uparrow \downarrow\rangle$. There are two electrons on the $\pi$-orbital.

Each state in this set is also an eigenvector of "electrons" number operator and "holes" number operator at the site $x$ :

$$
a_{x}^{\dagger} a_{x}\left|n_{x}, m_{x}\right\rangle=m_{x}\left|n_{x}, m_{x}\right\rangle ; \quad b_{x}^{\dagger} b_{x}\left|n_{x}, m_{x}\right\rangle=n_{x}\left|n_{x}, m_{x}\right\rangle ; \quad n_{x}, m_{x}=0,1 .
$$

Let us choose "vacuum" state as $|\downarrow \cdot\rangle$ with $n_{x}=0, m_{x}=0$ (there is no difference for Monte Carlo method what state is called as "vacuum"). Then $|\uparrow \downarrow\rangle$ with $n_{x}=1, m_{x}=0$ is "electron" excitation, $|\uparrow \cdot\rangle$ with $n_{x}=1, m_{x}=1$ is "electron+hole" state, and $|\cdot\rangle$ with $n_{x}=0, m_{x}=1$ is "hole" excitation. These vectors are eigenstates of charge operator with eigenvalues $q_{x}=n_{x}-m_{x}$. As seen from the above, there are two states with the same charges (equal to " 0 "). One of them is the "vacuum" state $|\downarrow \cdot\rangle$, and the other is the "electron+hole" state $|\uparrow \cdot\rangle$. There is also one state with charge "-1" $(|\downarrow \uparrow\rangle)$ and one state with charge " $+1 "(|\cdot\rangle)$.

Now we can define the state of the whole system $|S\rangle$ as an antisymmetrized product of oneelectron states: $|S\rangle=\left|\left\{n_{x}\right\}\left\{m_{x}\right\}\right\rangle$, where $\left\{n_{x}\right\}$ and $\left\{m_{x}\right\}$ are the distributions of occupation numbers for "electrons" and" holes" respectively at the whole lattice.

Let us discuss the meaning of three different parts of the full Hamiltonian (메). The values of electron-electron interaction potentials for suspended graphene one can see in [W]. The energy scale in hopping part of the Hamiltonian is defined by the value of $\kappa=2.7 \mathrm{eV}$. The energy scale of interaction part is defined by the Coulomb potential which is $9.3 \mathrm{eV}$ in the case of on-site interaction. It is 3 times larger than the hopping parameter $\kappa$. The eigenstates of particle number operator are also eigenstates of Hubbard and Coulomb terms of Hamiltonian $(\mathbb{L} . \mathbb{C})$ but not of the $H_{\kappa}$ term. Because of this fact and the fact that interaction part of the Hamiltonian gives possibly the main contribution to the energy, we will neglect $H_{\kappa}$. We can improve this method further by perturbation theory if necessary.

So the partition function has the form:

$$
Z=\sum_{\{n\}\{m\}} e^{-\frac{1}{T} H(\{n\}\{m\})}=\sum_{\{n\}\{m\}} \exp \left\{-\frac{1}{2 T}\left(\sum_{x} V_{x x}\left(n_{x}-m_{x}\right)^{2}+\sum_{x \neq y} V_{x y}\left(n_{x}-m_{x}\right)\left(n_{y}-m_{y}\right)\right)\right\}
$$


The average value of an observable quantity can be easily calculated if the eigenstates of particles number operators are also eigenstates of the operator of this observable. In this case, its average value can be represented as:

$$
\begin{aligned}
& \langle O\rangle=\frac{\sum_{\left\{q_{x}\right\}} O\left(\left\{q_{x}\right\}\right) e^{-\beta H} \prod_{x}\left(1+\delta_{q_{x}, 0}\right)}{\sum_{\left\{q_{x}\right\}} e^{-\beta H}}, \\
& H=\frac{1}{2}\left(\sum_{x} V_{x x}\left(q_{x}\right)^{2}+\sum_{x \neq y} V_{x y}\left(q_{x}\right)\left(q_{y}\right)\right),
\end{aligned}
$$

where $\beta=1 / T$, and $\left(1+\delta_{q_{x}, 0}\right)$ corresponds to the existence of two different states with $q_{x}=0$.

\section{Simple model: we take into account only interactions in the 1st coordination radius}

Let us consider the model with the following partition function

$$
Z=\sum_{\left\{q_{x}\right\}} \prod_{x}\left(1+\delta_{q_{x}, 0}\right) \exp ^{-\beta H\left(\left\{q_{x}\right\}\right)}, \text { where } H\left(\left\{q_{x}\right\}\right)=\frac{1}{2}\left(V_{00} \sum_{x} q_{x}^{2}+V_{01} \sum_{x, \rho_{b}} q_{x} q_{x+\rho_{b}}\right) .
$$

Only interaction with neighbouring sites is included into the Hamiltonian.

The first term in Hamiltonian corresponds to the interaction between electrons at one lattice site. The second term corresponds to the Coulomb interaction between the nearest neighbours. The vectors $\rho_{b}, b=1,2,3$ connect the nearest sites with each other.

The relation between $V_{00}$ and $V_{01}$ is the key quantity which defines the behaviour of the model. Let us consider the system at large $\beta$, when the ground state plays the main role in the partition function. We will consider firstly the limit $V_{00} \gg V_{01}$. In this case the self energy contribution, which corresponds to the on-site interaction term, dominates the interaction between neighbouring sites. The configuration with zero charges $q_{x}=0$ at all sites is the ground state of the system. In the opposite case $V_{00} \ll V_{01}$ the second term in the Hamiltonian (R.d)will dominate the first term and the ground state is the configuration where sites at different sublattices acquire opposite charges: $q_{x}=-q_{x+\rho_{b}}$. This situation is illustrated at the figure $\mathbb{W}$, where different charges are marked with different colors. Every term $q_{x} q_{x+\rho_{b}}$ gives a negative contribution into the energy. This contributions leads to the negative energy of such "chiral domain" in the limit $V_{01} \gg V_{00}$ and this configuration becomes a ground state because the zero charges configuration has zero energy. So, there is a critical value of $V_{00}^{c}$ where one ground state is replaced by another one.

The value of $V_{00}^{c}$ at large $\beta$ can be found from the following considerations. Zero energy of the "chiral domain" configuration marks the point of the phase transition. We can calculate the "chiral domain" energy per one lattice cell. One graphene lattice cell contains two sites and tree links between sites. The condition of zero energy per lattice cell can be written as follows: $V_{00}=3 \cdot V_{01}$.

It is impossible to find the critical $V_{00}^{c}$ analytically if we take into account finite temperature and interaction at all radii. So, we'll use Monte-Carlo to study this problem further in the next section. 


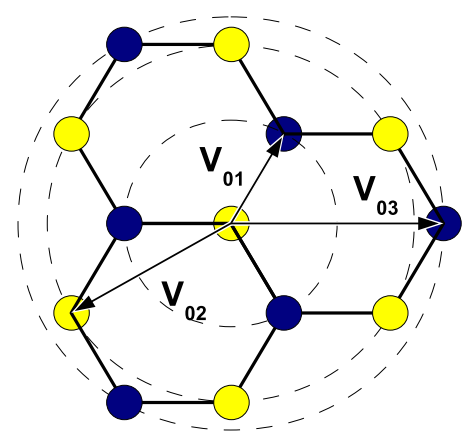

Figure 1: The system with charge separation.

\section{Monte Carlo investigation of the phase diagram}

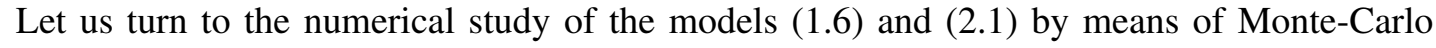
method. We have used the heat bath algorithm for our calculations, taking into account double weight of the states with zero charge $q_{x}=0$. We used hexagonal lattice with $36 \times 36$ sites and periodical boundary conditions.

We study the following observable to mark the phase transition:

$$
\langle O\rangle=\left\langle q_{x} q_{x+\rho_{b}}\right\rangle
$$

This average is very convenient for the detection of the transition between two vacua. Indeed, this quantity is close to zero in case disordered system. Otherwise, it is equal to -1 in the chiral domain phase, because $q_{x}$ and $q_{x+\rho_{b}}$ belong to different sublattices and have opposite charges.

We generated 100 statically independent configurations for every value of $V_{00}$ and $\beta=1 / T$. We changed the value of $V_{00}$ and hence the ratio between $V_{01}$ and $V_{00}$. The values of $V_{x y}$ at large distances correspond to ordinary Coulomb interaction. Its strength is defined by the $V_{01}$ potential. So we vary the value of $V_{00}$ potential leaving the other constant.

At the beginning, we consider the simple model (2. 1 ) where only the nearest neighbours interact with each other. The phase transition is marked by the maximum of derivation $\frac{\partial\langle O\rangle}{\partial T}$. The phase diagram is shown in the figure $\square$. There are tree different curves that corresponds to three different starts of Markov process during Monte-Carlo calculation. The bottom curve corresponds to start from configuration filled with zero charges. The top curve corresponds to chiral domain starting configuration. The curve between them corresponds to start from a special type of configuration: half of the lattice is filled with chiral domain and the rest of the lattice is filled with zero charges. The results of Monte Carlo algorithm shouldn't depend on the initial configuration for infinite thermalization time (infinite length of the Markov process). But in practice it's impossible to provide infinite time for thermalization. That is why we use that peculiar configuration for start. In this case, the long termalization is not necessary. We can only measure the change of the domain volume to determine the phase which will be a result of the thermalization process.

The accuracy of this method can be confirmed by the correspondence between low-temperature extrapolation presented in the the figure $\square$ and the theoretical value of the critical on-site interaction potential $V_{00}^{c}=3 \cdot V_{01}=16.5 \mathrm{eV}$. 


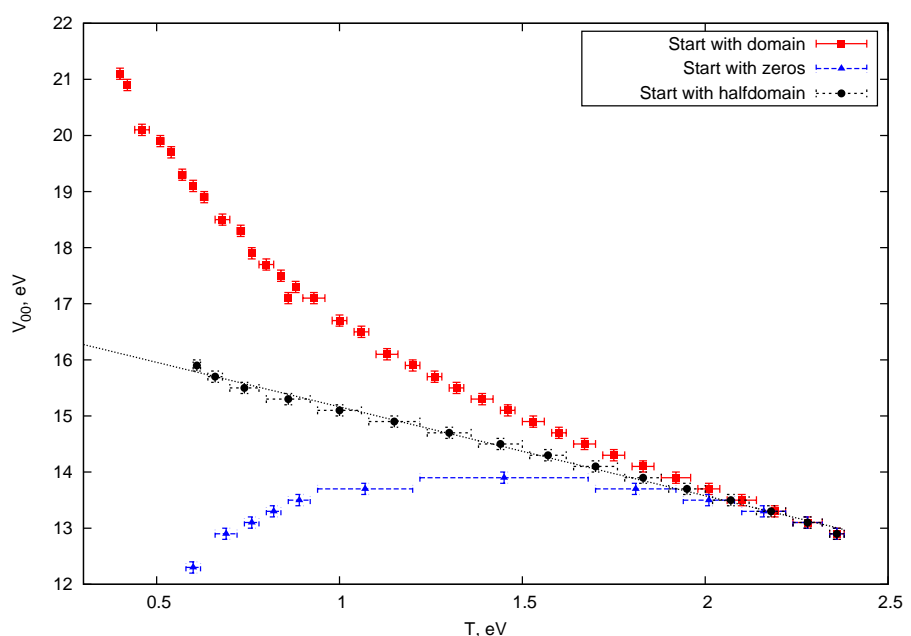

Figure 2: Phase diagram in the plane $\left(T, V_{00}\right)$ for the model which takes into account only interaction of the nearest neighbours. Three different kinds of initial configurations for termalization were used in the simulations.

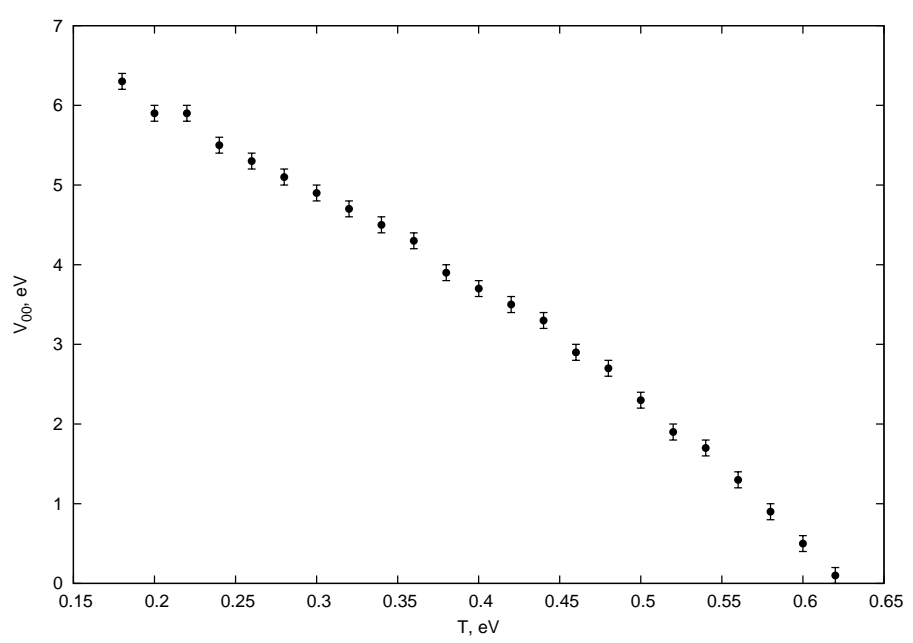

Figure 3: Phase diagram in the plane $\left(T, V_{00}\right)$ for the model with long range interaction. Start from halfdomain configuration.

Now let us investigate the system with interaction at all coordination radii. In this case we again use the start from lattice that is half-filled with domain. We detect the change of the chiral domain volume. If it becomes smaller, the domain melt, so the system turns into plasma. If domain grows, the systems turns into the phase with ordered charges. The result one can see in figure [3]. The comparison of figures $\square$ and $\mathbb{B}$ allows to trace the influence of Coulomb interaction at large coordination radii.

\section{Discussions and conclusion}

We investigated the phase diagram in the plane $\left(T, V_{00}\right)$ for the excitonic phase transition at hexagonal lattice. The main observation is that the value of the on-site interaction $V_{00}$ strongly 
influences the ground state of the system. Decreasing of $V_{00}$ can lead to the change of the system's ground state from zero charges configuration to the configuration with spontaneous charge separation between sublattices. The last phase is a dielectric with nonzero excitonic condensate. In the real graphene the $\sigma$ - orbital screening decreases the on-site potential $V_{00}$. But this screening is not enough strong for the phase transition even at zero temperature. The phase diagram strongly depends also on the interaction at large distances. So the real physical situation is a combination of two effects: the on-site interaction screened by $\sigma$ - orbitals and long range Coulomb interaction. The phase transition is very sensitive to both these factors. We obtained that free graphene is a conductor even at zero temperature. In order to achieve the dielectric state with nonzero excitonic condensate, the $\sigma$ - orbital screening should be stronger or something should block the Coulomb interaction between electrons at large distances.

\section{References}

[1] D. T. Son, Quantum critical point in graphene approached in the limit of infinitely strong Coulomb interaction, Phys. Rev. B75 (2007) 235423.

[2] D. V. Khveshchenko, Ghost Excitonic Insulator Transition in Layered Graphite, Phys. Rev. Lett. 87(2001) 246802.

[3] J. E. Drut and T. A. Lähde, Is Graphene in Vacuum an Insulator?, Phys. Rev. Lett. 102 (2009) 026802.

[4] W. Armour, S. Hands, and C. Strouthos, Monte Carlo simulation of the semimetal-insulator phase transition in monolayer graphene, Phys. Rev. $\mathbf{B 8 1}$ (2010) 125105.

[5] P. V. Buividovich, E. V. Luschevskaya, O. V. Pavlovsky, M. I. Polikarpov and M. V. Ulybyshev, Numerical study of the conductivity of graphene monolayer within the effective field theory approach, Phys. Rev. B86 (2012) 045107.

[6] A. S. Mayorov, D. C. Elias, I. S. Mukhin, S. V. Morozov, L. A. Ponomarenko, K. S. Novoselov, A. K. Geim, R. V. Gorbachev, How close can one approach the Dirac point in graphene experimentally?, Nano Lett. 12 (2012) 4629.

[7] M. V. Ulybyshev, M. I. Katsnelson, P. V. Buividovich and M. I. Polikarpov, Monte Carlo Study of the Semimetal-Insulator Phase Transition in Monolayer Graphene with a Realistic Interelectron Interaction Potential, Phys. Rev. Lett. 111 (2013) 056801.

[8] R. C. Brower, C. Rebbi, and D. Schaich, Hybrid Monte Carlo simulation of graphene on the hexagonal lattice, PoS LAT2011 (2012) 056, ArXiv:1204.5424.

[9] P. V. Buividovich and M. I. Polikarpov, Monte-Carlo study of the electron transport properties of monolayer graphene within the tight-binding model, Phys. Rev. B86 (2012) 245117.

[10] G. W. Semenoff, Condensed-Matter Simulation of a Three-Dimensional Anomaly, Phys. Rev. Lett. 53 (1984) 2449.

[11] T. O. Wehling, E. Sasioglu, C. Friedrich, A. I. Lichtenstein, M. I. Katsnelson, S. Blugel, Strength of effective Coulomb interactions in graphene and graphite, Phys. Rev. Lett. 106 (2011) 236805. 